\title{
In vivo temperature dependency of molybdenum and vanadium nitrogenases activity in the heterocystous cyanobacteria Anabaena variabilis
}

\section{Supporting Information.}

\author{
Romain Darnajoux $^{1,2,3^{*}}$, Robert Bradley ${ }^{2,3}$, Jean-Philippe Bellenger ${ }^{1,3^{*}}$ \\ ${ }^{1}$ Département de Chimie, Université de Sherbrooke, Sherbrooke, QC J1K 2R1, Canada \\ ${ }^{2}$ Département de Biologie, Université de Sherbrooke, Sherbrooke, QC J1K 2R1, Canada \\ ${ }^{3}$ Centre Sève, Université de Sherbrooke, Sherbrooke, QC J1K 2R1, Canada \\ \# Current address: Department of Geosciences, Princeton University, Princeton, NJ 08544, USA \\ Corresponding author: \\ Jean-philippe.bellenger@usherbrooke.ca \\ romaind@princeton.edu
}

Phone: 819-821-7014

Fax: 819-821-8017

ORCID: RD: 0000-0002-4996-0067, JPB: 0000-0001-6842-4561

Content: 13 pages containing 3 Supplementary Methods, 3 Supplementary Figures, 3 Supplementary Tables. 


\section{Supplementary Methods}

Supplementary Methods S1: Equivalence between enzyme activity and increase in ethylene partial pressure in ARACAS experiment.

Because $\sim 99 \%$ of ethylene is found in the headspace at all times during our experiments, we can equate the partial volume of ethylene gas in the headspace with the total mol of ethylene produced to obtain main text Eq [3].

$$
v(T)=\left(\frac{d[E t]}{d t}\right)_{T} \approx\left(\frac{d p p b v E t}{d t}\right)_{T} \times \frac{n_{\text {gas } t o t}}{V_{\text {media }}} \times 10^{9}
$$

With $n_{\text {gas }}$ tot the total number of mole in the headspace (mol), $p p b v_{E t}$ the volume fraction of ethylene in the headspace (ppbv), $V_{\text {media }}$ the volume of liquid in our system (L). As $n_{\text {gas }}$ tot, and $V_{\text {media }}$ remain mostly constant during our experiments, both terms will cancel out when using relative rate as in main text $\mathrm{Eq}[3]$ 
Supplementary Methods S2: Effect of temperature on substrate saturation.

According to main text Eq [2], Sat $(T)=f\left([A c]_{T}, K_{M}(T)\right)$, so we can express the total derivative of $S a t(T)$ with respect to $T$ as a function of the partial derivative of $K_{M}(T)$ and $[A c]_{T}$ to yield Eq [S1]

$$
\begin{aligned}
{\left[\frac{d(\text { Sat })}{d T}\right] } & =\frac{\partial \operatorname{Sat}(T)}{\partial[A c]} \times \frac{d[A c]}{d T}+\frac{\partial \operatorname{Sat}(T)}{\partial K_{M}} \times \frac{d K_{M}}{d T} \\
& =\frac{K_{M}}{\left(K_{M}+[A c]\right)^{2}} \frac{d[A c]}{d T}-\frac{[A c]}{\left(K_{M}+[A c]\right)^{2}} \frac{d K_{M}}{d T}
\end{aligned}
$$

Equation [S1] shows that when Km increase with temperature, saturation decrease (mind the minus sign). It also highlights that when substrate concentration decreases with temperature $\frac{d[A c]}{d T}$, (as due to a change in solubility), it is dependant on the $K_{M}$ of the enzyme. Hence, the effect of substrate change with temperature is $20 \%$ to $200 \%$ stronger on V-Nase than on MoNase. 
Supplementary Methods S3: Extrapolation of relative enzymatic activity of $V$ vs. Mo Nase toward nitrogen reduction.

According to Main text Eq 3, we can express the relative activity toward nitrogen reduction at any temperature $\left(v_{N 2}(\mathrm{~T})\right)$ of $\mathrm{V}-\mathrm{Nase}$ over Mo-Nase as a function of their respective specific activity $\left(\mathrm{k}_{2}(\mathrm{~T})\right)$, substrate saturation $\left(\operatorname{Sat}_{\mathrm{N} 2}(\mathrm{~T})\right)$, and substrate-independent enzyme concentrations ([Nase](T)), and re-organized into Eq. S2 by applying this relationship at $22.5^{\circ} \mathrm{C}$ :

$$
\begin{aligned}
& \frac{v_{V, N 2}(T)}{v_{M o, N 2}(T)}=\frac{\left[\frac{k_{2, N 2}(T)}{k_{2, N 2}\left(22.5^{\circ} \mathrm{C}\right)}\right]_{V}}{\left[\frac{k_{2, N 2}(T)}{k_{2, N 2}\left(2.5^{\circ} \mathrm{C}\right)}\right]_{M o}} \times \frac{k_{2, V, N 2}\left(22.5^{\circ} \mathrm{C}\right)}{k_{2, M o, N 2}\left(22.5^{\circ} \mathrm{C}\right)} \times \frac{\operatorname{Sat}_{V, N 2}(T)}{\operatorname{Sat}_{M o, N 2}(T)} \times \frac{[\text { Nase }]_{V}(T)}{[N a s e]_{M o}(T)} \\
& \frac{v_{V, N 2}(T)}{v_{M o, N 2}(T)}=\frac{\left[\frac{k_{2, N 2}(T)}{k_{2, N 2}\left(22.5^{\circ} \mathrm{C}\right)}\right]_{V}}{\left[\frac{k_{2, N 2}(T)}{k_{2, N 2}\left(22.5^{\circ} \mathrm{C}\right)}\right]_{M o}} \times \frac{v_{V, N 2}\left(22.5^{\circ} \mathrm{C}\right)}{v_{M o, N 2}\left(22.5^{\circ} \mathrm{C}\right)} \times \frac{\left[\frac{\operatorname{Sat}_{N 2}(T)}{\operatorname{sat}_{N 2}\left(22.5^{\circ} \mathrm{C}\right)}\right]_{V}}{\left[\frac{\operatorname{Sat} t_{N 2}(T)}{\operatorname{sat}_{N 2}\left(22.5^{\circ} \mathrm{C}\right)}\right]_{M o}} \times \frac{\left[\frac{[\text { Nase }(T)}{\left[\text { Nase }\left(22.5^{\circ} \mathrm{C}\right)\right.}\right]_{V}}{\left[\frac{[\text { Nasee }(T)}{[\text { Nase }]\left(22.5^{\circ} \mathrm{C}\right)}\right]_{M o}}
\end{aligned}
$$

At low temperature $\left(\mathrm{T}<22^{\circ} \mathrm{C}\right)$, we can write Eq S3 based on main text Eq 5, and noticing that the difference between $\Delta \mathrm{HV}_{\mathrm{v}}$ and $\Delta \mathrm{H}_{\mathrm{Mo}}$ at $22.5^{\circ} \mathrm{C}$ and $\Delta \mathrm{Cp}$ and $\Delta \mathrm{Cp}_{\mathrm{Mo}}$ are similar for acetylene and nitrogen reduction (See Main Text Table 1)

$$
\begin{aligned}
\frac{\left[\frac{k_{2, N 2}(T)}{k_{2, N 2}\left(22.5^{\circ} \mathrm{C}\right)}\right]_{V}}{\left[\frac{k_{2, N 2}(T)}{k_{2, N 2}\left(22.5^{\circ} \mathrm{C}\right)}\right]_{M o}} & =\frac{e^{\left(-\frac{\Delta H_{V}\left(22.5^{\circ} \mathrm{C}\right)}{R}\left(\frac{1}{T}-\frac{1}{22.5}\right)+\frac{\Delta c_{p_{V}}}{R}\left[\ln \left(\frac{T}{22.5}\right)+\frac{T_{b}}{T}-1\right]\right)}}{e^{\left(-\frac{\Delta H_{M o}\left(22.5^{\circ} \mathrm{C}\right)}{R}\left(\frac{1}{T}-\frac{1}{22.5}\right)+\frac{\Delta C_{p_{M o}}}{R}\left[\ln \left(\frac{T}{22.5}\right)+\frac{T_{b}}{T}-1\right]\right)}} \\
& =e^{\left(-\frac{\left.\Delta H_{V}\left(22.5^{\circ} \mathrm{C}\right)-\Delta H_{M o}\left(22.5^{\circ} \mathrm{C}\right)\right)}{R}\left(\frac{1}{T}-\frac{1}{22.5}\right)+\frac{\left(\Delta C_{p_{V}}-\Delta C_{p_{M o}}\right)}{R}\left[\ln \left(\frac{T}{22.5}\right)+\frac{T_{b}}{T}-1\right]\right)} \\
& \approx \frac{\left[\frac{k_{2, A c}(T)}{k_{2, A c}\left(22.5^{\circ} \mathrm{C}\right)}\right]_{V}}{\left[\frac{k_{2, A c}(T)}{k_{2, A c}\left(22.5^{\circ} \mathrm{C}\right)}\right]_{M o}}
\end{aligned}
$$

At high temperatures $\left(\mathrm{T}>22^{\circ} \mathrm{C}\right)$, Mo and $\mathrm{V}$ have similar activation energies, independent of isozymes and thus Eq S3 stays true and can be approximate to 1 . 
We can further express the ratio of relative specific activity of isozyme toward acetylene according to main text $\mathrm{Eq} 1$ as a function of isozyme activities, isozyme concentrations, and isozyme saturations (Eq S4):

$$
\frac{\left[\frac{k_{2, A c}(T)}{k_{2, A c}\left(22.5^{\circ} \mathrm{C}\right)}\right]_{V}}{\left[\frac{k_{2, A c}(T)}{k_{2, A c}\left(22.5^{\circ} \mathrm{C}\right)}\right]_{M o}}=\frac{\left[\frac{v_{A c(T)}}{v_{A c}\left(22.5^{\circ} \mathrm{C}\right)}\right]_{V}}{\left[\frac{v_{A c}(T)}{v_{A c}\left(22.5^{\circ} \mathrm{C}\right)}\right]_{M o}} \times \frac{\left[\frac{[\text { Nase }(T)}{\left[\text { Nase }\left(22.5^{\circ} \mathrm{C}\right)\right.}\right]_{M o}}{\left[\frac{[\text { Nase }(T)}{[\text { Nase }]\left(22.5^{\circ} \mathrm{C}\right)}\right]_{V}} \times \frac{\left[\frac{\operatorname{Sat}_{A c}(T)}{\operatorname{Sat} A c\left(22.5^{\circ} \mathrm{C}\right)}\right]_{M o}}{\left[\frac{\operatorname{Sat} A c(T)}{\operatorname{Sat} A c\left(22.5^{\circ} \mathrm{C}\right)}\right]_{V}}
$$

By injecting Eq S5 and S6 into Eq S4, we obtain Eq S7, which simplifies into main text Eq 6.

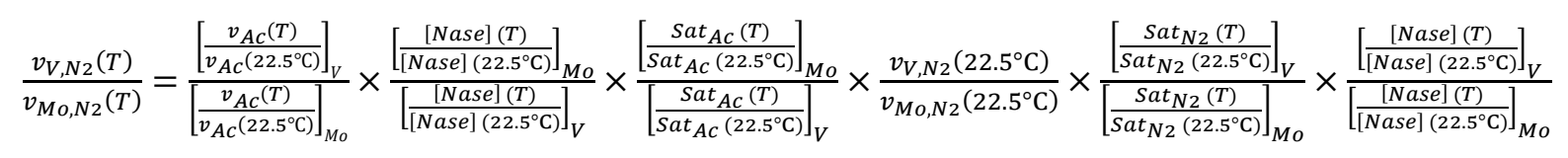

$$
\begin{aligned}
& \frac{v_{V, N 2}(T)}{v_{M o, N 2}(T)}=\frac{\left[\frac{v_{A C}(T)}{v_{A c}\left(22.5^{\circ} \mathrm{C}\right)}\right]_{V}}{\left[\frac{v_{A c}(T)}{v_{A c}\left(22.5^{\circ} \mathrm{C}\right)}\right]_{M o}} \times \frac{\left[\frac{\operatorname{Sat}_{A c}(T)}{\operatorname{Sat}_{A c}\left(22.5^{\circ} \mathrm{C}\right)}\right]_{M o}}{\left[\frac{S a t_{A c}(T)}{\operatorname{Sat}_{A c}\left(22.5^{\circ} \mathrm{C}\right)}\right]_{V}} \times \frac{\left[\frac{\operatorname{Sat}_{N 2}(T)}{\operatorname{Sat}_{N 2}\left(22.5^{\circ} \mathrm{C}\right)}\right]_{V}}{\left[\frac{S a t_{N 2}(T)}{\operatorname{Sat}_{N 2}\left(22.5^{\circ} \mathrm{C}\right)}\right]_{M o}} \times \frac{v_{V, N 2}\left(22.5^{\circ} \mathrm{C}\right)}{v_{M o, N 2}\left(22.5^{\circ} \mathrm{C}\right)}
\end{aligned}
$$




\section{Figure S1}

A

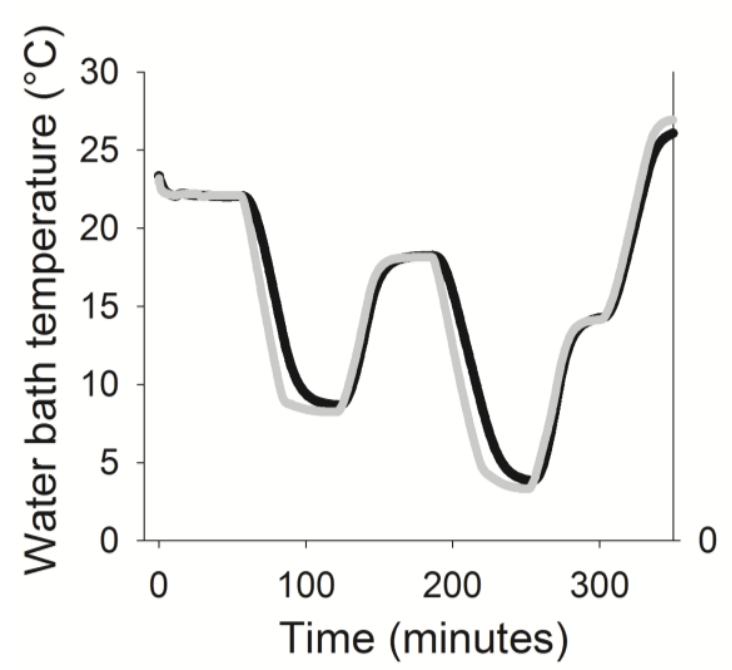

C

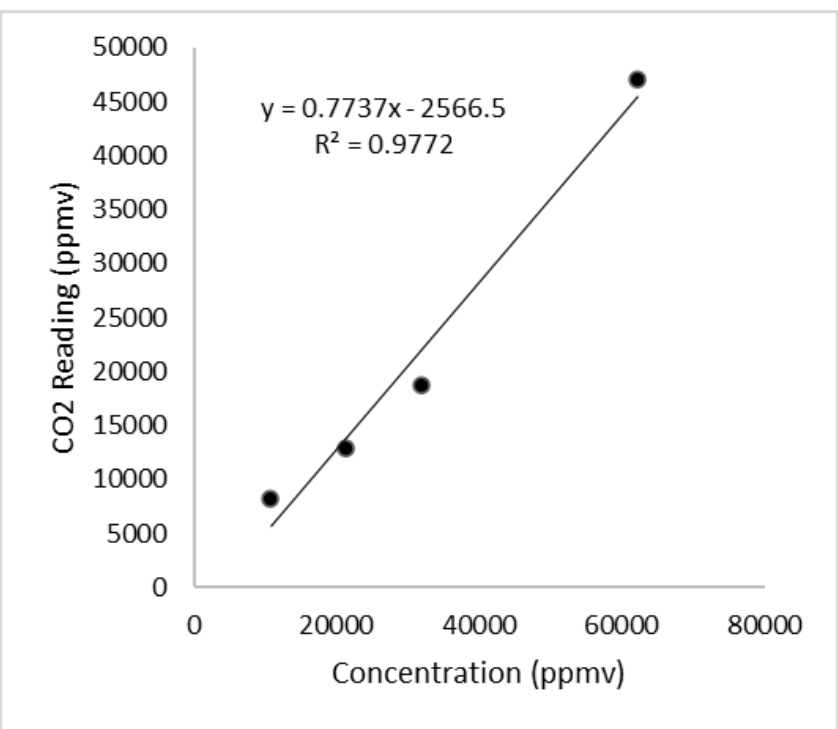

B

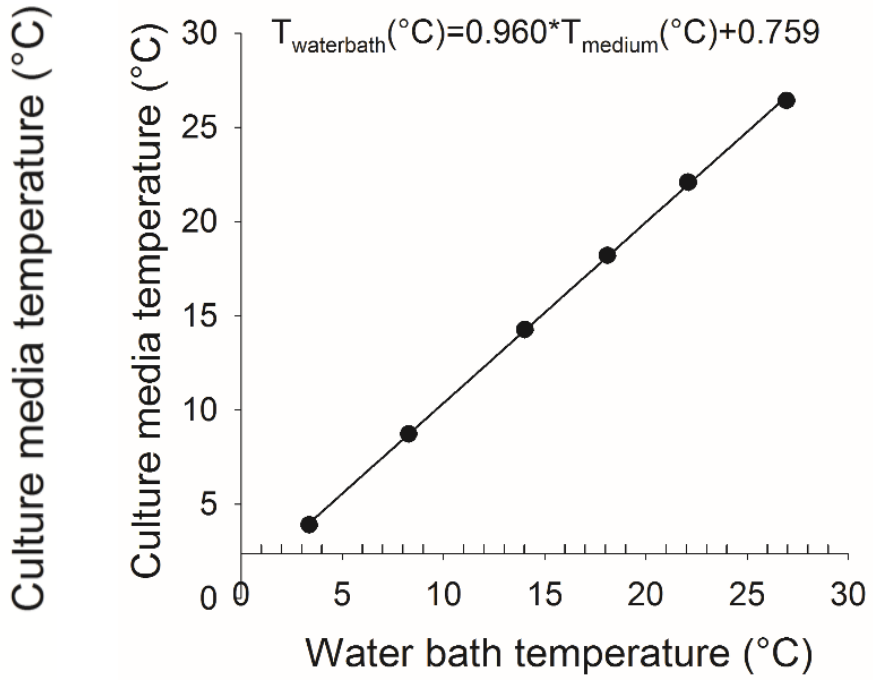

D

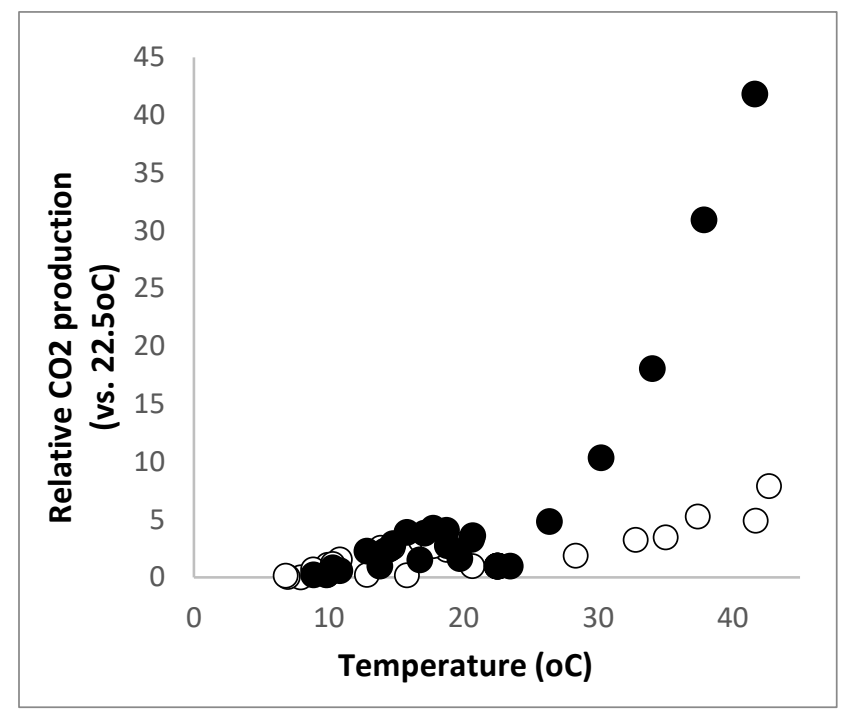

Figure S1: Dynamic response $(A)$ and calibration $(B)$ of temperature within the incubation vessel and linearity of the $\mathrm{G1106} \mathrm{CO}_{2}$ channel $(\mathrm{C})$ and relative $\mathrm{CO}_{2}$ production with temperature (D). Water bath temperature (grey curve) and culture media temperature (black curve). (A), and calibration of temperature of media inside the incubation vessel and temperature of the water bath outside the incubation vessel (B). The linearity of the response was determined by adding incremental volumes of $100 \% \mathrm{CO}_{2}$ into the incubation vessel. In D, close symbols are Mo-Nase, and open symbols are V-Nase. 


\section{Figure S2}

A

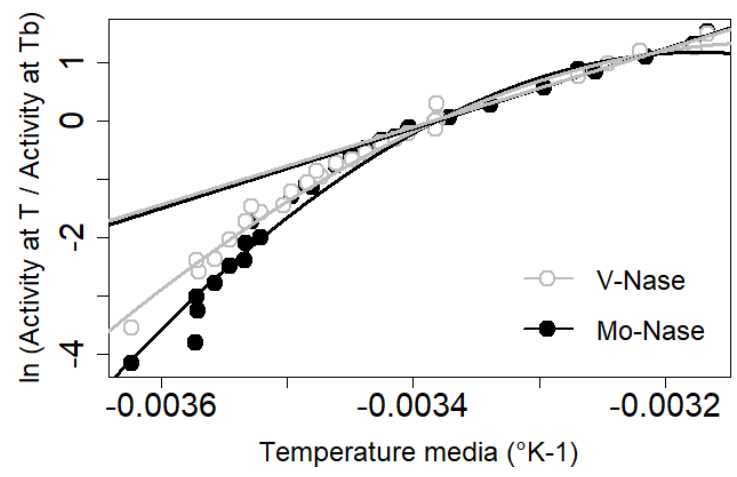

C

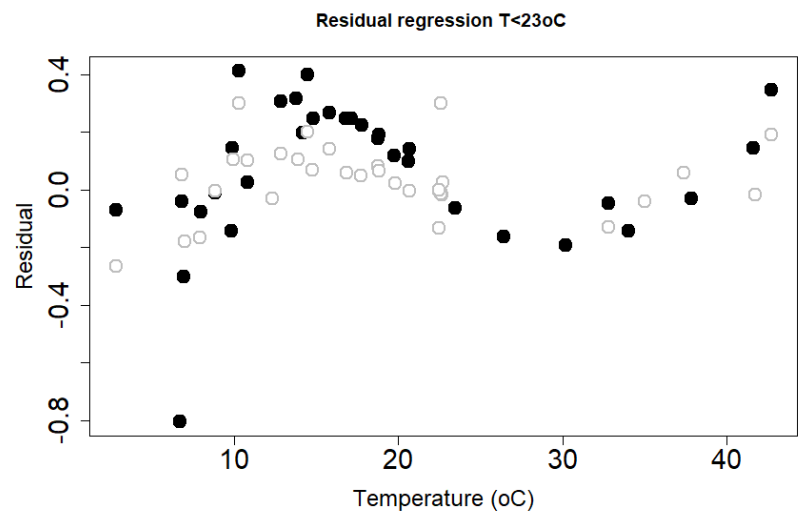

B
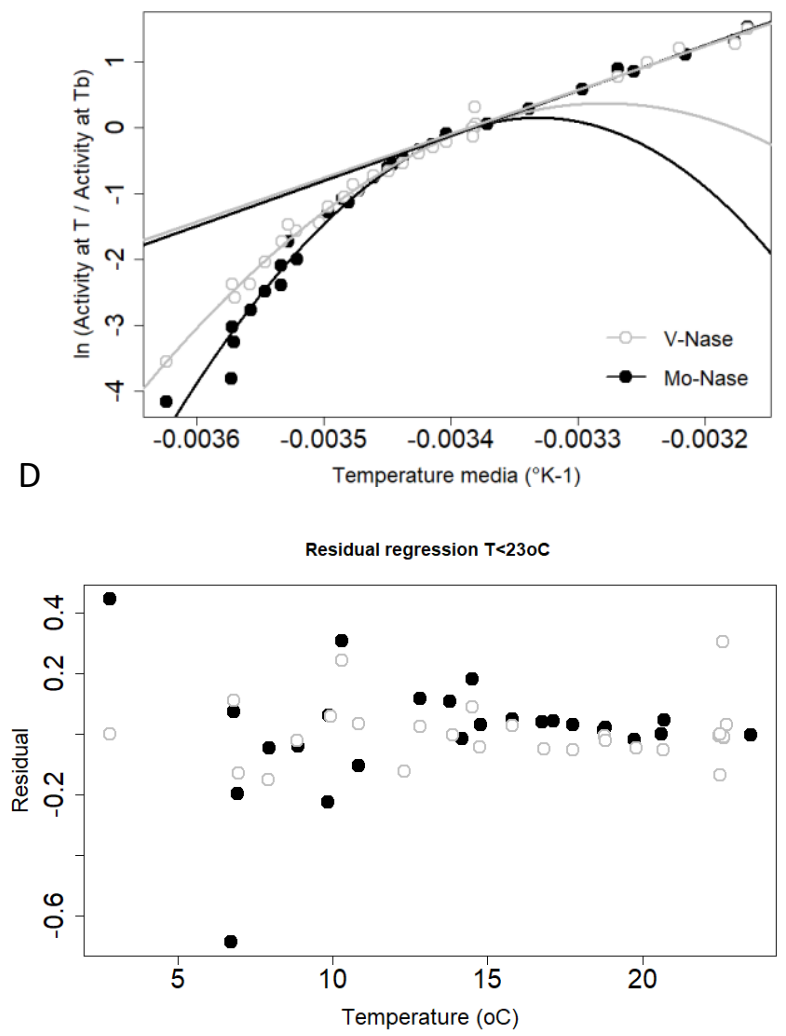

Figure S2: Regression's continuity and residuals analysis for regression of Figure 3A with continuity between the non-linear and linear regression for Mo and V-Nase relative activity data $(A \& B)$ and residual of the non-linear regression portion $(C \& D)$. Non-linear regression was conducted on all points $(\mathrm{A} \& \mathrm{C})$ or only on points for $\mathrm{T}<23^{\circ} \mathrm{C}(\mathrm{B} \& \mathrm{D})$. Analysis of the residuals clearly shows that the non-linear regression is not performing well for the full dataset (see the sinusoidal residual) vs. the point for $\mathrm{t}<23^{\circ} \mathrm{C}$. Visual inspection of panel B shows the continuity between non-linear and linear regression is around $22^{\circ} \mathrm{C}\left(0.0034{ }^{\circ} \mathrm{K}^{-1}\right)$. 
Figure S3

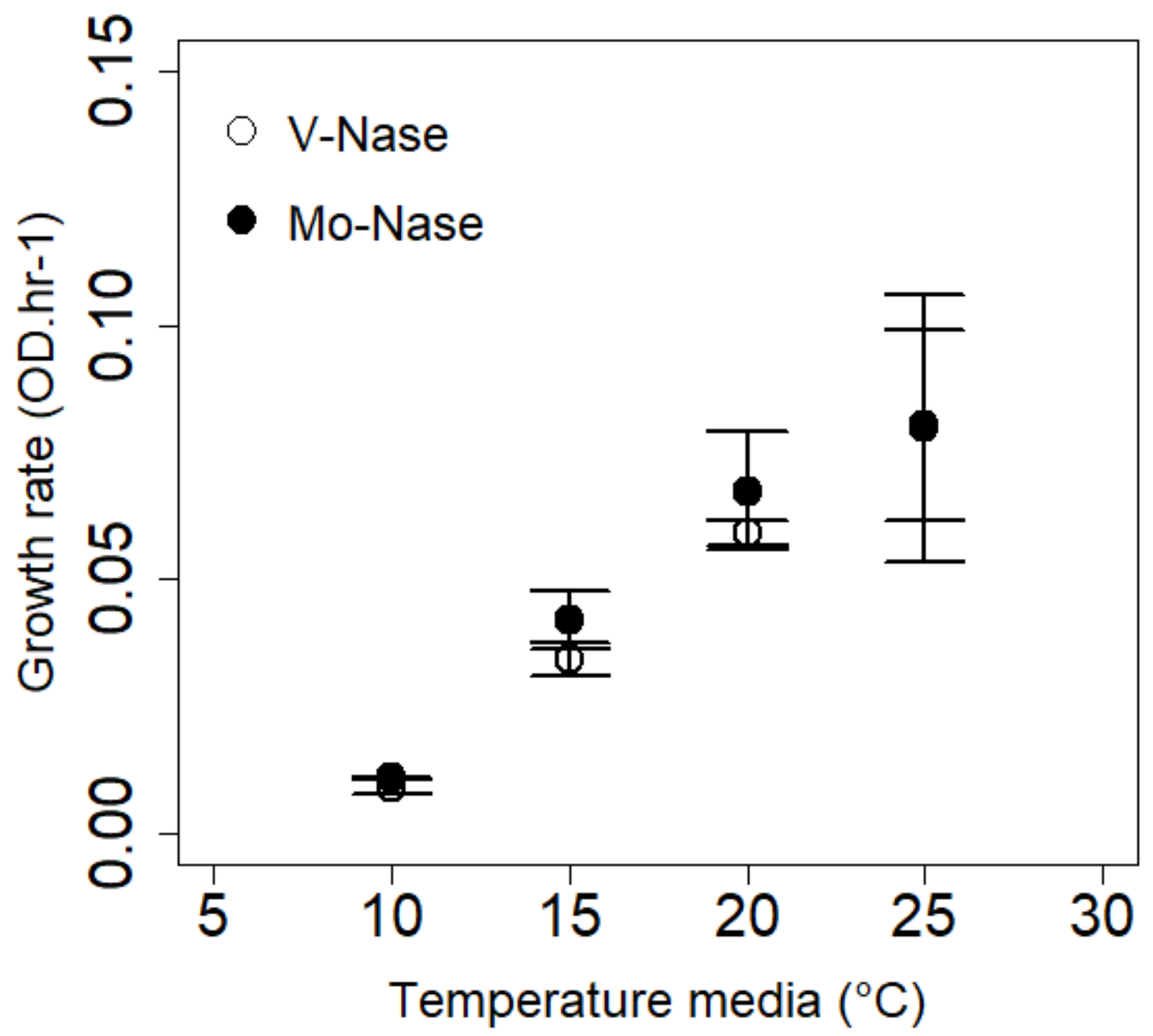

Figure S3: Growth of Anabaena variabilis as a function of temperature. Evolution of growth

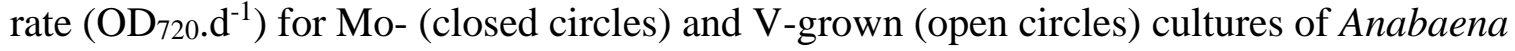
variabilis. Error bars are $\mathrm{SD}$ and $\mathrm{n}=3$ except at $15^{\circ} \mathrm{C}(\mathrm{n}=6$ and 18 for Mo and $\mathrm{V}$, respectively) 
TABLE S1: Summary of experimental design for \#Exp 1 (Figure 1) and changes in activity and biomass (as chlorophyll a and $\mathrm{OD}_{720}$ over the experiments per replicate.

\begin{tabular}{|c|c|c|c|c|c|c|c|c|c|c|c|}
\hline \# EXP & $\begin{array}{c}\text { Temperature } \\
\text { program }\end{array}$ & $\begin{array}{l}\mathrm{OD}_{720} \\
\text { start }\end{array}$ & $\begin{array}{l}\mathrm{OD}_{720} \\
\text { end }\end{array}$ & $\begin{array}{l}\text { Chl A } \\
\text { start }\end{array}$ & $\begin{array}{l}\text { Chl A } \\
\text { end }\end{array}$ & $\begin{array}{l}\text { Activity } \\
\text { start }\end{array}$ & $\begin{array}{l}\text { Activity } \\
\text { end }\end{array}$ & $\begin{array}{c}\text { Length } \\
\text { Experiment }\end{array}$ & $\begin{array}{l}\text { Change } \\
\text { OD }_{720}\end{array}$ & $\begin{array}{l}\text { Change } \\
\text { Chl A }\end{array}$ & $\begin{array}{l}\text { Change } \\
\text { activity }\end{array}$ \\
\hline & ${ }^{\circ} \mathrm{C}$ & - & - & ug. $\mathrm{mL}^{-1}$ & ug. $\mathrm{mL}^{-1}$ & ppb. $\min ^{-1}$ & ppb.min ${ }^{-1}$ & $\mathrm{~h}$ & $\%$ & $\%$ & $\%$ \\
\hline Mo_1 & $22 / 23 / 19 / 16 / 13 / 9 / 6$ & ND & ND & ND & ND & 813 & 781 & $5: 35$ & ND & ND & -4 \\
\hline Mo_2 & $22 / 19 / 16 / 13 / 9 / 6 / 22$ & 0.765 & 0.86 & 3.185 & 3.185 & 346 & 347 & $6: 07$ & 12 & 0 & 0 \\
\hline Mo_3 & $22 / 18 / 15 / 12 / 7 / 22$ & 0.925 & 0.96 & 3.455 & 3.085 & 109 & 103 & $6: 13$ & 4 & -11 & -5 \\
\hline Mo_4 & $\begin{array}{c}22 / 20 / 17 / 14 \\
/ 10 / 8 / 22\end{array}$ & 1.49 & 1.22 & 3.69 & 2.615 & 167 & 142 & $5: 41$ & -18 & -29 & -15 \\
\hline Mo_5 & $\begin{array}{c}22 / 26 / 30 / 34 / 38 / 42 / \\
22\end{array}$ & 1.055 & 1.105 & 3.1875 & 3.69 & 189 & 234 & $5: 31$ & 5 & 16 & 24 \\
\hline Mo_6 & $\begin{array}{c}22 / 18 / 14 / 10 / 6 / 2 / 12 \\
/ 22 * / 33 / 43 / 22 * *\end{array}$ & 0.554 & ND & 2.304 & ND & 251 & 356 & $7: 56$ & ND & ND & $* 42 / * *-14$ \\
\hline$V_{-1}$ & $22 / 19 / 16 / 13 / 9 / 6$ & 0.546 & 0.745 & ND & 2.415 & 158 & 105 & 7:11 & 36 & ND & $-34^{1}$ \\
\hline$V_{-} 2$ & $22 / 18 / 15 / 12 / 7 / 22$ & 0.63 & 0.705 & 2.215 & 2.615 & 62 & 62 & $5: 52$ & 12 & 18 & 0 \\
\hline V_3 & $22 / 20 / 17 / 14 / 10 / 8$ & 0.85 & 1.135 & 3.02 & 2.985 & 153 & 93 & $5: 48$ & 34 & -1 & -39 \\
\hline$V_{-} 4$ & $22 / 28 / 35 / 42 / 22$ & 1.35 & $2.815^{2}$ & 4.16 & 6.26 & 158 & 166 & $9: 37$ & $109^{2}$ & 50 & 5 \\
\hline$V_{-} 5$ & $\begin{array}{l}22 / 18 / 14 / 10 / 6 / 2 / 12 \\
/ 22 * / 32 / 37 / 43 / 22 * *\end{array}$ & 0.5 & ND & 2.362 & ND & 96 & 131 & $\begin{array}{c}10: 56 \\
(7 h: 4 h)\end{array}$ & ND & ND & $* 36 / * *-13$ \\
\hline
\end{tabular}

${ }^{1}$ Data was excluded as activity was still increasing when the experiment was stopped.

${ }^{2}$ Data was excluded as culture volume was clearly reduced due to evaporation. 
TABLE S2: Summary of changes in biomass and activity of the courses of our experiment.

Change during the experiment (\%)

\begin{tabular}{llllll}
\hline Length (h) & $\mathrm{OD}_{720}$ & Chla & Activity & Total Biomass & Total \\
\hline
\end{tabular}

\begin{tabular}{|c|c|c|c|c|c|c|}
\hline \multicolumn{7}{|c|}{$\mathrm{T}<22.50 \mathrm{C}$} \\
\hline Average & $6: 08$ & 13 & -5 & -10 & 5 & 0 \\
\hline SD & $0: 32$ & 20 & 17 & 15 & 20 & 20 \\
\hline $\mathrm{n}$ & 6 & 6 & 5 & $6^{*}$ & 11 & $17^{*}$ \\
\hline \multicolumn{7}{|c|}{$\mathrm{T}>22.50 \mathrm{C}$} \\
\hline Average & $8: 30$ & 57 & 33 & 27 & 24 & 25 \\
\hline SD & $2: 20$ & 73 & 25 & 16 & 24 & 18 \\
\hline $\mathrm{n}$ & 4 & 2 & 2 & 4 & $3 *$ & $7^{*}$ \\
\hline
\end{tabular}

*one value was removed from the estimates 
TABLE S3: Summary of statistical models and results.

\begin{tabular}{|c|c|c|c|c|c|c|c|}
\hline \multirow[t]{12}{*}{ Figure 2C } & Group & Statistical model & function (package) & Variables & Estimate & SE & $\operatorname{Pr}(>|t|)$ \\
\hline & All & $\mathrm{Km} \sim$ Nase $+\mathrm{T}+\varepsilon$ & Im (stats) & Intercept & -0.5 & 0.4 & 0.224 \\
\hline & & & & Temperature & 0.12 & 0.02 & $<0.001$ \\
\hline & & & & Nase (V-Nase) & 7.9 & 0.3 & $<0.001$ \\
\hline & V-Nase & $\mathrm{Km} \sim$ correction $/ \mathrm{T}+\varepsilon$ & Im (stats) & Intercept & 4.8 & 0.5 & $<0.001$ \\
\hline & & & & Correction & 4.4 & 0.7 & 0.001 \\
\hline & & & & Uncorrected: $\mathrm{T}$ & 0.24 & 0.03 & $<0.001$ \\
\hline & & & & Corrected : T & 0.05 & 0.03 & 0.150 \\
\hline & Mo-Nase & $\mathrm{Km} \sim$ correction $/ \mathrm{T}+\varepsilon$ & Im (stats) & Intercept & -0.51 & 0.10 & 0.003 \\
\hline & & & & Correction & 0.42 & 0.15 & 0.027 \\
\hline & & & & Uncorrected: $\mathrm{T}$ & 0.121 & 0.006 & $<0.001$ \\
\hline & & & & Corrected : $\mathrm{T}$ & 0.100 & 0.006 & $<0.001$ \\
\hline \multirow[t]{11}{*}{ Figure $3 A$} & & & & Variables & SS (df) & F-Value & $P(>F)$ \\
\hline & $\begin{array}{c}\text { All } \\
(T<220 C)\end{array}$ & Eq. [5] vs. Eq.[5] | Nase & anova_nlslist (nlshelper) & Eq. [5] & $0.28(6)$ & & \\
\hline & & & & Eq. [5] | Nase & $0.02(4)$ & & \\
\hline & & & & ANOVA & $0.26(2)$ & 27.909 & $<0.001$ \\
\hline & & & & Variables & Estimate & SE & $\operatorname{Pr}(>|t|)$ \\
\hline & $\begin{array}{c}\text { V-Nase } \\
(T<220 \mathrm{C})\end{array}$ & Eq.[5] & nlsList (nlstools) & $\mathrm{DH}(\mathrm{Tb})$ & 121691 & 4144 & 0.001 \\
\hline & & & & DCp & -8617 & 701 & 0.007 \\
\hline & $\begin{array}{l}\text { Mo-Nase } \\
(T<220 C)\end{array}$ & Eq.[5] & nlsList (nlstools) & $\mathrm{DH}(\mathrm{Tb})$ & 147577 & 1600 & $<0.001$ \\
\hline & & & & DCp & -13739 & 270 & $<0.001$ \\
\hline & $\begin{array}{c}\text { All } \\
(T>220 C)\end{array}$ & $\begin{array}{c}\text { In }(\mathrm{k} 2 \text { rel }) \sim \mathrm{T} \text { inv. } / \\
\text { Nase }+\varepsilon\end{array}$ & Im (stats) & T inv. & -4796 & 148 & $<0.001$ \\
\hline & & & & Tinv : Nase (V-Nase) & 3 & 7 & 0.76 \\
\hline
\end{tabular}




\begin{tabular}{|c|c|c|c|c|c|c|c|}
\hline \multirow[t]{9}{*}{ Figure 3B } & & & & Variables & SS (df) & F-Value & $P(>F)$ \\
\hline & \multirow[t]{3}{*}{ All } & $\begin{array}{c}\ln (\mathrm{CO} 2 \text { rel. }) \sim \text { Nase }+\mathrm{T} \\
\text { inv. }+\varepsilon\end{array}$ & \multirow[t]{3}{*}{ anova (stats) } & Nase $+\mathrm{T}$ inv. & $0.188(7)$ & & \\
\hline & & $\begin{array}{c}\text { vs. In (CO2 rel.) } \sim \text { Nase } \\
* T \text { inv }+\varepsilon\end{array}$ & & Nase * $\mathrm{T}$ inv. & $0.188(6)$ & & \\
\hline & & & & ANOVA & $0.0002(1)$ & 0.0063 & 0.939 \\
\hline & & & & Variables & Estimate & SE & $\operatorname{Pr}(>|t|)$ \\
\hline & \multirow[t]{2}{*}{ V-Nase } & \multirow[t]{4}{*}{$\mathrm{CO} 2 \sim \mathrm{T}$ inv. $+\varepsilon$} & \multirow[t]{2}{*}{ Im (stats) } & Intercept & 24.693 & 7.225 & 0.042 \\
\hline & & & & T inv. & 7198.656 & 2246.913 & 0.049 \\
\hline & \multirow[t]{2}{*}{ Mo-Nase } & & \multirow[t]{2}{*}{ Im (stats) } & Intercept & 27.259 & 5.442 & 0.015 \\
\hline & & & & T inv. & 7421.395 & 1691.675 & 0.022 \\
\hline \multirow[t]{5}{*}{ Figure 4B } & & & & Variables & SS (df) & F-Value & $P(>F)$ \\
\hline & \multirow[t]{4}{*}{ All } & \multirow[t]{4}{*}{ Growth $\sim$ Nase ${ }^{*} \mathrm{~T}+\varepsilon$} & \multirow[t]{4}{*}{ anova (stats) } & Nase & $0.3346(1)$ & 16.9239 & $<0.001$ \\
\hline & & & & $\mathrm{T}$ & $6.5365(1)$ & 330.5781 & $<0.001$ \\
\hline & & & & Nase:T* & $0.0777(1)$ & 3.9312 & 0.055 \\
\hline & & & & Residuals & $\begin{array}{c}0.5734 \\
(29)\end{array}$ & & \\
\hline
\end{tabular}

SE: Standard error, SS: residual sum of square 
